\title{
Muséologies
}

Les cahiers d'études supérieures

\section{La muséologie est un livre : entretien avec François Mairesse}

\section{Alessandra Mariani}

Volume 6, numéro 1, 2012

URI : https://id.erudit.org/iderudit/1011536ar

DOI : https://doi.org/10.7202/1011536ar

Aller au sommaire du numéro

Éditeur(s)

Association Québécoise de Promotion des Recherches Étudiantes en Muséologie (AQPREM)

ISSN

1718-5181 (imprimé)

1929-7815 (numérique)

Découvrir la revue

Citer ce document

Mariani, A. (2012). La muséologie est un livre : entretien avec François Mairesse. Muséologies, 6(1), 137-149. https://doi.org/10.7202/1011536ar

Tous droits réservés (C Association Québécoise de Promotion des Recherches Étudiantes en Muséologie (AQPREM), 2012
Ce document est protégé par la loi sur le droit d'auteur. L’utilisation des services d'Érudit (y compris la reproduction) est assujettie à sa politique d'utilisation que vous pouvez consulter en ligne.

https://apropos.erudit.org/fr/usagers/politique-dutilisation/ 
Dialogue deux

La muséologie est un livre :

entretien avec François Mairesse

Alessandra Mariani 
Dirigé par André Desvallées et François Mairesse, le Dictionnaire encyclopédique de muséologie est paru au printemps 2011 aux éditions Armand Colin. Il rassemble 500 termes et 21 articles encyclopédiques présentant l'étendue du champ muséal dans une optique pratique, historique et théorique. Premier ouvrage du genre dans l'univers francophone, tout a néanmoins été mis en ouvre pour refléter la diversité des idées et des acceptions de ce champ véhiculées dans plusieurs autres langues. De la collection à l'exposition, de la gestion à l'éducation, de la recherche à la communication, le Dictionnaire encyclopédique de muséologie interroge, documente et commente le système muséal.

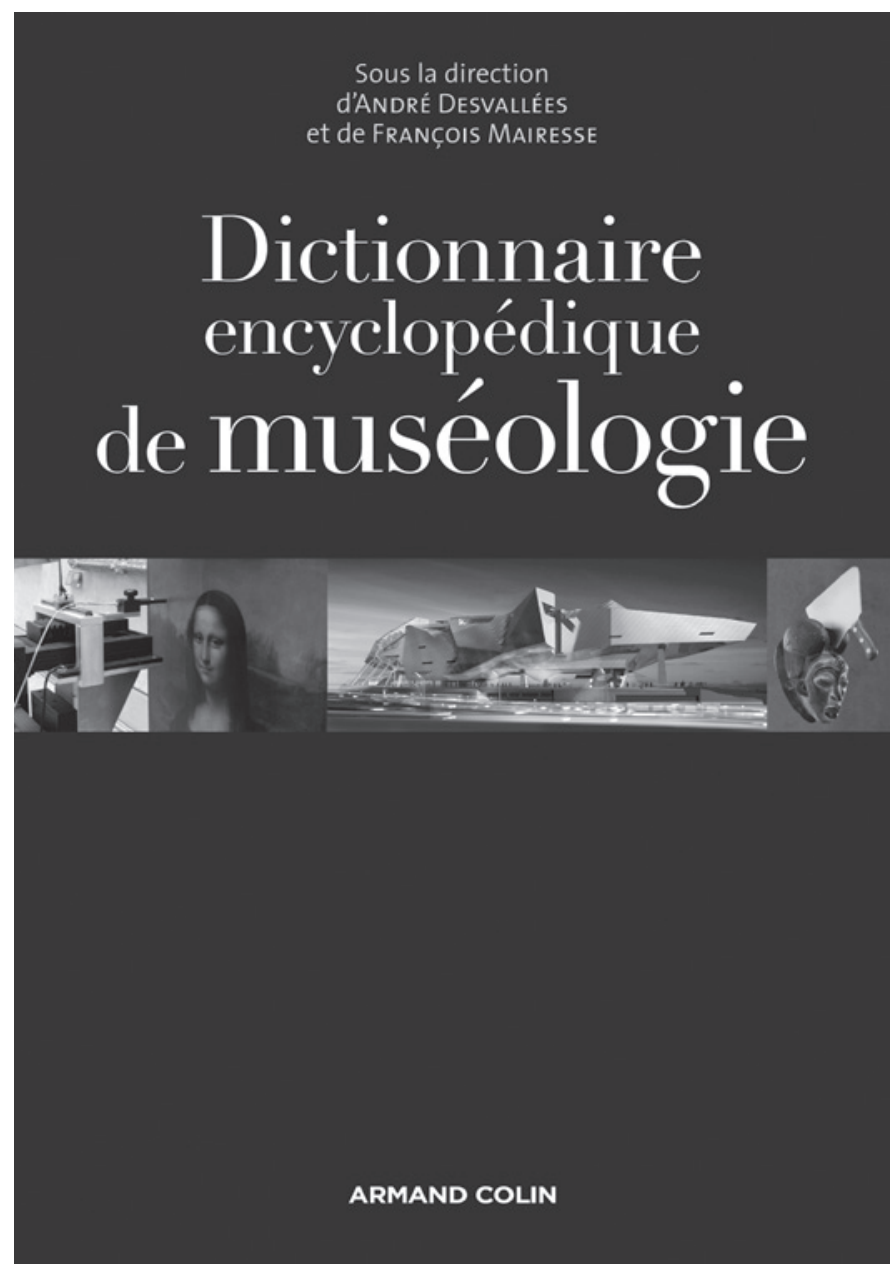


François Mairesse est professeur d'économie de la culture à l'Université de Paris 3 . Il enseigne la muséologie à l'École du Louvre. Après un mastère en gestion et un mastère en histoire de l'art à l'Université Libre de Bruxelles, il a obtenu son doctorat en 1998 de la même université et son habilitation à diriger des recherches à l'Université Jean Moulin à Lyon en 2008. Il a successivement travaillé pour le Fonds national de la recherche scientifique, le Cabinet du Ministre Président de la Communauté française de Belgique et, de 2002 à 2010 , au Musée royal de Mariemont (Belgique) dont il a assumé la direction. Il a enseigné la muséologie à l'Université Jean Moulin (Lyon 3) de 1999 à 2010, ainsi qu'à l'Université de Liège. Il a publié plusieurs articles et ouvrages de muséologie, dont, parmi les plus récents: Le Dictionnaire encyclopédique de muséologie (codirigé avec André Desvallées), Paris, Armand Colin, 2011 ; Le musée hybride, Paris, La Documentation française, 2010 ; L'inaliénabilité des collections de musées en question (dirigé), Morlanwelz, Musée royal de Mariemont, 2009 ; Pourquoi (ne pas) aller au musée (écrit avec Bernard Deloche), Lyon, Aléas, 2008 ; Mariemont, capitale du don: des Warocqué aux Amis de Mariemont, Morlanwelz, Musée royal de Mariemont, 2007 ; Vers une redéfinition du musée? (codirigé avec André Desvallées), Paris, L'Harmattan, 2007 ; Le droit d'entrer au musée, Bruxelles, Labor, 2005. 
François Mairesse, vous avez, avec André Desvallées, récemment publié le Dictionnaire encyclopédique de muséologie. Pouvez-vous nous raconter la genèse de ce projet?

FM Ce projet est, pourrait-on dire, pratiquement aussi vieux que l'ICOFOM, le Comité international pour la muséologie, qui a été créé en 1977. Déjà à cette époque plusieurs personnes se sont réunies autour d'un projet de rédiger un grand traité de muséologie. Georges-Henri Rivière, fondateur du Musée national des arts et traditions populaires à Paris, s'était impliqué dans cette aventure dès les tout débuts. Malgré le fait que le projet ne se soit pas réalisé à ce moment-là, il était relativement abouti. Il faut comprendre que le programme était énorme (quatre ou cinq volumes): on en parle d'ailleurs encore dans la revue de l'ICOFOM - les MuWop (Museological Working Papers) - comme quelque chose de réalisable, même si plusieurs circonstances ont empêché son aboutissement. Il est donc demeuré à l'État de projet. Entretemps, les MuWop ont été remplacés par la revue Icofom Study Series, mais il restait toujours à publier cet ouvrage estampillé ICOFOM.

C'est dans cette perspective qu'au début des années 1990 le président de l'ICOFOM, Martin Schärer ${ }^{1}$, relance l'idée de présenter un certain nombre de concepts de muséologie qui justement seraient édités par l'ICOFOM. André Desvallées, conservateur général honoraire du patrimoine (Musées de France) est alors chargé du projet : il commence par publier officieusement quelques articles dont certains paraissent dans la revue Publics et musées (aujourd'hui Culture et Musées). Bien qu'il ait développé quelques termes et qu'il ait reçu de nombreux encouragements, personne ne s'était réellement avancé pour le suivre dans cette entreprise. En 2000 ils en étaient toujours au stade de projection avec un certain nombre de définitions qui reflétaient des aboutissements très différents. Le mot "patrimoine" par exemple, bien développé, avait fait l'objet de plusieurs articles d'André. Deux personnes se sont proposées pour agrandir le cercle: la philosophe argentine Norma Rusconi, qui avait offert d'écrire un certain nombre de textes (mais qui est malheureusement décédée quelques années plus tard), et moi-même; j'avais déjà proposé un article à André et je me suis progressivement joint au projet. Nous avons alors tenté d'imaginer une nouvelle structure pour le volume, réalisable en quelques années, qui aurait l'avantage d'être composé de deux parties: une formant le dictionnaire et l'autre, l'encyclopédie, avec une vingtaine d'entrées importantes, comme l'avait été "patrimoine", reliées à un certain nombre d'autres termes qui étaient déjà définis de façon plus brève par André dans son lexique paru dans le Manuel de muséographie ${ }^{2}$. C'est dans cette perspective que nous avons progressivement commencé à rédiger la définition d'un certain nombre de mots, dont "muséologie» et "muséalisation".

1 Martin Schärer est directeur de l'Alimentarium (le Musée de l'alimentation), à Vevey en Suisse; il a été viceprésident à l'ICOFOM pendant de très nombreuses années. 2 Sous la direction de Marie-Odile DE BARY et Jean-Michel TOBELEM. Paris : Séguier, 2000. 
Les choses se sont ensuite accélérées : si nous avions colligé suffisamment d'information, nous nous sommes rapidement rendu compte qu'il serait plus intéressant de poursuivre ce travail en équipe, de constituer une assise plus importante et de rassembler autour du projet un cercle un peu plus grand de personnes qui avaient une réelle propension et un intérêt pour l'écriture. Il s'agissait en fait de personnes que nous connaissions bien et qui étaient proches de l'ICOFOM puisqu'elles en étaient membres.

À l'occasion du Congrès de 2009 de l'ICOFOM à Liège, qui était consacré au projet du dictionnaire, nous avons été honorés par la présence du directeur général du Conseil international des musées (ICOM), Julien Anfruns. Je dois avouer que lors de la première journée plusieurs membres avaient exprimé quelques réticences quant à la production du dictionnaire encyclopédique; ils se disaient qu'avec l'arrivée de cet ouvrage nous allions "figer " les choses. Ce genre de réaction s'est surtout manifesté parmi ceux qui ne l'avaient ni vu, ni lu. Nous avons produit le dictionnaire dans la perspective de concrétiser, de réunir, de colliger un nombre de savoirs, et surtout de donner une image, un objet concret à la muséologie, qui déterminent le fait que ce champ n'est pas qu'études de publics, gestion des œuvres, collections, etc. Il s'agit d'un champ beaucoup plus vaste, plus global et plus porteur, avec une véritable dynamique.

Après son introduction, Julien Anfruns est venu nous dire que ce que nous faisions était important et nous avons ainsi reçu un support moral important de l'ICOM. Ensuite, par l'intermédiaire d'André Gob, professeur en muséologie à l'Université de Liège, j'ai contacté l'éditeur Armand Colin qui s'est montré très rapidement intéressé. Colin avait déjà développé ce créneau de la muséologie à travers différents ouvrages et sa maison d'édition souhaitait élargir ce type de publications. En ce qui a trait à l'édition même de l'ouvrage, je tenais beaucoup au principe de base d'une sorte de teaser, une information qui pouvait se diffuser de manière très large. L'idée de départ était que la partie des encadrés puisse être distribuée gratuitement, notamment par le biais d'Internet, nous appuyant sur une logique selon laquelle des produits gratuits permettent une diffusion plus vaste du projet. Armand Colin a trouvé qu'il s'agissait d'un bon concept et a accepté de superviser l'ensemble. L'ICOM, l'ICOFOM et le Musée royal de Mariemont ont participé à la publication des concepts clés de muséologie: édités pour la première fois en français, en anglais, en espagnol et en chinois, afin de faire parler de l'ouvrage, ils ont été réunis dans un petit livret qui a été distribué aux participants de la $22^{\mathrm{e}}$ conférence de l'ICOM à Shanghai en 2010. À bien des égards, il s'agissait évidemment d'une manœuvre stratégique; non seulement pour présenter le livre, mais aussi et surtout pour présenter une vision francophone de la muséologie, qui n'est de toute évidence pas la même partout. C'est dans cette perspective que plusieurs nous ont montré leur intérêt à obtenir une version des concepts clés traduite dans d'autres langues, et nous avons ainsi développé des projets de traduction (par exemple en tchèque, en letton, en japonais) qui sont pour le moment assez avancés. Nous avons produit des versions 
téléchargeables sur le site de l'ICOM dans les quatre langues officielles: sous l'onglet "Que faisons-nous ", on trouve dans la liste du menu ces concepts clés de muséologie.

\section{Vous avez fait appel à plusieurs collaborateurs pour constituer cet ouvrage. Pouvez-vous rapidement nous les présenter ainsi que leur contribution à cette recherche?}

FM J'ai eu la chance d'accompagner pendant de très nombreuses années André Desvallées et j'en suis devenu en quelque sorte le disciple. Déjà quand je rédigeais ma thèse, bien qu'il n'était pas mon directeur, il était présent; il enseignait alors à l'École du Louvre. C'est au cours de rencontres successives dans des colloques que je me suis familiarisé avec sa pensée et que je fus impressionné par ses positions sur les avancées théoriques du champ muséologique. Je me suis rendu compte qu'il était de toute évidence une personnalité emblématique de la muséologie française. Nous avons par la suite sympathisé et constaté que nous partagions les mêmes idées spirituelles. J'ai proposé à André certaines personnes qui pourraient contribuer à l'élaboration du dictionnaire - il s'agissait bien entendu de personnes avec lesquelles nous avions des affinités parce qu'il aurait été impossible de mener à bien ce projet dans la discorde. Il fallait forcément un esprit de cohésion et de coopération; il fallait des gens compétents qui acceptaient de coopérer.

L'initiateur est donc André Desvallées et je l'ai suivi. Très rapidement, il y a tout un groupe qui s'est rallié autour du projet, dont trois Québécois. Deux d'entre eux ont écrit des textes: Yves Bergeron, directeur des études supérieures en muséologie à l'Université du Québec à Montréal (UQAM) (plusieurs articles) et Raymond Montpetit, professeur associé au département d'histoire de l'art à l'UQAM (un article); le troisième, Philippe Dubé, directeur du programme de DESS en muséologie à l'Université Laval a contribué à la partie dictionnaire. La partie touchant l'éducation et celle sur les professions dérivées ont été confiées à Serge Chaumier, professeur en muséologie à l'Université d'Artois, qui aussi est assez proche de notre groupe. Une personne fédératrice a été importante dans ce projet, Bernard Deloche, philosophe et muséologue, professeur émérite à l'Université Jean Moulin Lyon 3. Nous le connaissions par ses publications importantes, dont Muséologica (J. Vrin, 1985) et Le musée virtuel (Presses universitaires de France, 1999). C'est, à mon avis, la personne qui a la vision la plus structurée de la muséologie et qui proposait quelque chose d'extrêmement articulé de ce système. Nous étions vraiment heureux qu'il puisse participer à la définition d'un certain nombre de termes qu'il a lui-même développés, dont "muséal " (terme a priori un peu curieux pour le néophyte, mais qui permet de structurer le champ de la muséologie). Bernard Deloche est une figure extrêmement importante dans le domaine: j'espère que cette plate-forme permettra de mieux faire connaître ses écrits, d'une grande importance pour la muséologie.

Lorsqu'on se situe dans un travail de réflexion, on se trouve inévitablement dans une dimension philosophique : c'est clairement dans cette notion de muséal qu'est articulé tout le reste et c'est pour cette raison que Bernard Deloche est l'un des piliers de ce projet. Par ailleurs, 
Martin Schärer a travaillé sur l'éthique avec Bernard, et sur l'exposition avec André; et Noémie Drouguet, maître de conférences à l'Université de Liège, s'est penchée sur la même problématique. Il fallait forcément produire un ouvrage susceptible de fédérer des positions parfois différentes, présentées par des chercheurs reconnus tels que Peter van Mensch ou Susan Pearce: il s'agissait de rassembler et d'assembler plusieurs points de vue; de comprendre et de dresser une carte de tous ces éléments afin de montrer quelle était la place de l'un et de l'autre. La participation d'André Gob, qui a été très actif durant plusieurs réunions, a été importante pour cet aspect. Jean Davallon, directeur du programme international de muséologie à l'Université d'Avignon et des pays de Vaucluse a, lui aussi collaboré, avec Yves Bergeron, à l'article intitulé «Recherche».

\section{L'encyclopédie comporte 21 termes (ou articles ou concepts} ou essais) qui constituent les articles encyclopédiques, ainsi que d'un dictionnaire de muséologie. Comment en êtes-vous arrivés à déterminer ces concepts et pourquoi vous êtes-vous limités à ceux-ci?

FM Nous étions en 2007 et je dirigeais à l'époque le Musée royal de Mariemont; il y eut un premier séminaire où nous avons tracé un cahier des charges assez précis avec une dizaine de collaborateurs qui s'engageaient véritablement à rédiger les articles sur ces termes dans des délais préétablis. Nous nous sommes réunis avec toutes les personnes qui avaient été préalablement contactées pour le projet avec une première version des termes: après discussions et restructuration, nous en sommes arrivés à un consensus de 20 termes auquel nous en avons rajouté un autre, à la suite du colloque de Liège. Les ébauches de ces définitions ont par la suite été maintes fois retravaillées: nous nous étions fixé un délai de deux ans pour compléter le travail. Pour la première étape, tous les participants devaient avoir suffisamment développé leurs idées par écrit pour permettre de débattre de nombreux aspects. Plusieurs des éléments abordés devenaient des termes qui allaient aussi être rédigés de façon un peu plus brève. En somme, ce dictionnaire en devenir reposait sur celui qu'André Desvallées avait déjà pratiquement construit sept ou huit ans plus tôt sur 140 termes de muséologie (que j'ai évoqué plus haut). Cet ouvrage a été fort utile puisqu'il avait eu jusque-là une forte résonnance auprès de toute une série de publications qui en reprenaient les termes et les définitions.

Nous avons ensuite décidé d'organiser l'année suivante, à Liège, un colloque complet de l'ICOFOM où la version provisoire d'une vingtaine de termes serait présentée. L'idée était aussi qu'il fallait forcément que l'ensemble des membres du colloque soient d'accord avec notre démarche: nous souhaitions récolter leurs commentaires et leurs suggestions afin de tenir compte de toutes les réactions. Au même moment, nous voulions aussi discuter de la partie des encadrés, qui offrent la définition première et communément acceptée des spécialistes de chacun des termes. 
À partir de ces 20 concepts il s'agissait de proposer une structure générale, de définir des jalons qui délimitent l'ensemble du champ. Le nombre de concepts aurait pu être plus élevé, mais il s'agissait surtout d'arriver à concrétiser le projet dans des délais raisonnables. Il fallait aussi les limiter parce qu'il s'agissait de produire des articles vastes et importants comportant des renvois.

Un an plus tard a donc eu lieu le colloque annoncé, lors du XXXII symposium de l'ICOFOM, autour des 20 concepts que tous les participants avaient reçus à l'avance, afin qu'ils puissent réagir et ainsi nous permettre de récolter leurs commentaires. Une série de personnalités reconnues pour leur grande connaissance du champ (Jean Davallon, Raymond Montpetit, Pascal Griener, Michèle Antoine, Peter van Mensch) avaient accepté de lire trois, quatre, cinq termes et de donner un point de vue détaillé sur les textes. Peter van Mensch, professeur d'héritage culturel à la Amsterdam School of the Arts, avait reçu "muséologie » et "muséalisation "; Michèle Antoine, responsable des expositions à l'Institut royal des Sciences naturelles de Belgique a lu la partie sur les expositions; pour Pascal Griener, professeur à l'Institut d'Histoire de l'Art et de Muséologie de l'Université de Neuchâtel ce fut "musée» et "institution ", et ainsi de suite. Chacun était responsable de son petit domaine, parce que nous ne pouvions pas demander à tous de lire plus de 300 ou 400 pages et de réagir aux écrits. C'est à ce moment-là que Jean Davallon et Raymond Montpetit ont proposé des lectures extrêmement pertinentes et que nous avons réagi en leur demandant d'intégrer notre groupe de rédacteurs, ainsi qu'un $21^{\mathrm{e}}$ concept, celui de "médiation ", qui a été confié à Raymond. Nous ne voulions pas, comme je l'ai déjà avancé, une liste complètement fermée, mais nous ne souhaitions pas non plus nous retrouver avec une liste ouverte à tous vents. Ce n'est qu'un an plus tard, à la suite de remaniements, que l'on a pu finalement rendre le manuscrit. Parallèlement, André Desvallées et moi-même travaillions essentiellement sur la partie du dictionnaire. Des 140 termes que nous avions repris au départ, nous en sommes arrivés à 500. Nous avons bien entendu demandé l'avis judicieux de quelques experts, ce qui nous a beaucoup aidés. Jean-Jacques Ezrati, grand spécialiste de l'éclairage, a proposé beaucoup de termes spécifiques concernant ce domaine par exemple; Philippe Dubé a accepté de travailler sur l'ensemble du dictionnaire pour faire une dernière lecture globale en proposant un certain nombre de termes nouveaux aussi; Dominique Poulot, professeur d'histoire du patrimoine à l'Université de Paris 1 Panthéon-Sorbonne, a élaboré un article spécifique, tout comme Martin Schärer. Par ailleurs, Jean-Michel Tobelem, directeur d'Option Culture, a accepté de relire toutes les parties qui concernaient l'aspect gestion, Marie Cornu, spécialiste du droit patrimonial en France, a accepté de revoir d'un point de vue juridique un certain nombre de définitions afin qu'elles ne soient pas contradictoires, etc.

Pour chacun des 21 termes, il y a un encadré. Cette partie, comme je l'ai déjà expliqué, devait faire consensus. La partie encyclopédique est quand à elle présentée autour d'une partie historique et de l'ensemble du concept et du développement d'un certain nombre d'éléments 
prospectifs ou plus conflictuels, etc. Il s'agissait aussi de ne pas simplement s'attarder sur le passé, mais de démontrer, entre autres, quels étaient les enjeux. Par exemple, quand on parle d'architecture, on explique aussi un certain nombre de difficultés, de perspectives complexes; sous "patrimoine", on souligne qu'il s'agit d'une notion occidentale pouvant être réfutée dans d'autres pays. Cette partie a donc été laissée sous la responsabilité des auteurs, tandis que l'encadré n'est pas signé puisqu'il s'agit d'un travail collectif.

Chacun de ces articles présente la description d'un concept et l'ancre dans plusieurs registres (histoire, philosophie, économie, etc.). Pouvez-vous nous en dire davantage sur l'élaboration du plan de surface des articles?

FM Comme je le disais plus tôt, le plan général a été assez longuement débattu; nous l'avons constitué ainsi afin qu'il y ait une même logique dans les articles de base. Nous avons beaucoup insisté pour lui donner une voix qui soit la plus large possible, autant anglophone que francophone, et nous avons tenté - dans un certain nombre de cas - d'aborder la littérature muséologique hispanophone, ou allemande (par le biais de Martin Schärer), par exemple, et d'inclure la littérature des pays de l'Est qui, elle aussi, a un poids déterminant dans le domaine. Il ne s'agissait pas de rester entre Français, nous visions la plus grande exhaustivité possible. Nous n'avions pas de modèle préétabli: nous avons pensé au modèle de l'encyclopédie, parce que nous voulions faire système et proposer une certaine vision du monde à travers la muséologie. La logique générale sur laquelle nous étions tous d'accord était que la muséologie n'est pas une science mais une discipline en devenir. Nous ne voulions pas trancher ni créer d'étau autour du champ: il s'agissait de souligner le fait que la muséologie est un champ ouvert, recentré sur une perspective, une problématique qui est celle du «muséal ». L'idée était de démontrer qu'il s'agissait d'un domaine où les savoirs sont parfaitement transmissibles et enseignables.

Les encyclopédies ou dictionnaires destinés à la muséologie sont plutôt rares : vous mentionnez vous-même seulement deux ouvrages - le Dictionarium museologicum (ICOM, 1986) et le Lexique de muséologie, écrit par Jean Blanchet (Secrétariat d'État du Canada, 1989) - qui avaient été rédigés au cours des années 1980 , donc avant le vôtre. Est-ce que vous pourriez nous les présenter brièvement et expliquer comment le dictionnaire encyclopédique en muséologie vient combler leurs lacunes? FM C'est assez simple parce que même si le premier s'appelle Dictionarium, il s'agissait clairement d'un lexique précis et daté: si toute une série de termes n'y figurent pas, il avait néanmoins l'ambition de travailler sur le sujet. Il y a jusqu'à 20 traductions de chaque terme, ce qui est un travail très impressionnant et malheureusement peu connu, mais il n'y a pas de définition. Ces deux ouvrages sont les seuls documents traitant du champ muséal pur; il existe un dictionnaire anglophone intitulé Museum Wise: Workplace Words Defined ${ }^{3}$ qui traite des termes de façon beaucoup plus 
technique, mais l'encyclopédie était nécessaire pour traduire un mot par un autre. Il ne s'agissait pas du tout de faire un état des lieux, ni de définir des termes. Le dictionnaire encyclopédique de muséologie ne s'est d'ailleurs pas toujours appelé comme cela: on l'a nommé Thésaurus, puis Encyclopédie et c'est finalement le concept de dictionnaire encyclopédique qui nous a semblé le plus intéressant parce qu'il réunissait la partie encyclopédique avec la partie dictionnaire.

\section{Quelle sera l'étendue de diffusion de l'ouvrage: sera t-il traduit en plusieurs langues, comptez-vous développer une version numérique ou accessible en ligne?}

FM Je pense qu'il existe déjà une version accessible en ligne, commercialisée par Armand Colin. Nous avons eu la chance de bénéficier du concours du Centre national du livre parce que, pour un éditeur, il s'agit d'une entreprise assez risquée qui n'attirera pas nécessairement un marché très large. Il faut reconnaître que la muséologie n'est pas un très vaste champ, en regard de la sociologie par exemple, et c'est un ouvrage qui s'est révélé de plus en plus lourd. Le livre, qui devait compter autour de 300 à 400 pages, en fait maintenant 800. Ce n'était pas simple, mais en prenant ce risque, en croyant en ce créneau qu'est la muséologie, Armand Colin nous a offert un support extraordinaire. Depuis que les Presses universitaires de Lyon avaient mis un frein aux publications en 2002, L'Harmattan offrait quelques collections qui se prêtent au champ, mais il n'y avait plus vraiment de lieu pour publier. Armand Colin remplit maintenant bien ce rôle. Il y a d'ailleurs plusieurs maisons d'édition qui pourraient être intéressées par les traductions. La plus grande difficulté est justement la question de la traduction; aucun éditeur n'a pour l'instant accepté de prendre à sa charge ce type de frais; traduire 800 pages requiert un budget colossal, et cela nous incite à chercher une solution. La volonté de traduire en plusieurs langues est bien là, mais la traduction en anglais demeure une priorité. L'ICOFOM a reçu un petit budget de l'ICOM pour amorcer les travaux, mais la marge est grande entre les besoins réels et cette amorce. Il faudra réfléchir autrement, même si le temps joue pour nous puisque nous devons attendre pour constater le succès de ce dictionnaire. Il y a une volonté chez les Chinois, les Japonais (qui sont en train de traduire les concepts clés); c'est un élément très positif. Il y a de l'intérêt de la part des Italiens, comme de la part de certains pays de l'Amérique latine, mais soyons réalistes: il faut trouver des gens de bonne volonté qui ont, en plus, les connaissances nécessaires pour traduire. Nous souhaitons que ce document devienne une référence dans la francophonie, mais aussi à l'échelle mondiale, et qu'il soit, a fortiori, intégré dans l'espace anglo-saxon parce qu'il y a, à mon sens, des enjeux stratégiques pour une certaine vision de la muséologie. Je dis stratégique parce que si le Smithsonian avait réalisé un ouvrage similaire cela aurait été, de toute évidence, selon une autre vision de la muséologie. 


\section{Un ouvrage comme celui-ci est appelé à se transformer dans le temps. Comment voyez-vous son évolution et comment souhaitez-vous qu'elle soit réalisée?}

FM Ce qui est remarquable chez Armand Colin, c'est qu'on nous a déjà demandé de réfléchir assez rapidement à la deuxième édition. Ils ont constaté, par exemple, que les ouvrages d'André Gob en étaient à leur deuxième, voire leur troisième édition; ils savent bien qu'une entreprise comme celle-ci ne s'arrêtera pas à une seule version, qu'elle sera de toute évidence augmentée, d'autant plus qu'il est clairement indiqué dans le dictionnaire qu'il comporte des carences et que des améliorations sont possibles et souhaitées. Nous comprenons qu'une structure en évolution est nécessaire pour refléter ce champ et c'est pour nous très positif.

\section{L'ouvrage a été lancé une première fois au printemps 2011 : comment a-t-il été reçu?}

FM Il a été lancé au sein de l'ICOM, durant l'assemblée annuelle, et présenté par Julien Anfruns lui-même, en présence d'André Desvallées et d'Armand Colin. Rapidement l'ouvrage a été acquis par toutes les bibliothèques universitaires; les musées se rendent maintenant compte qu'il peut être intéressant. Nous souhaitons qu'il puisse être acquis par des gens qui ont parfois recours à la muséologie (par exemple des architectes, des scénographes ou des concepteurs d'expositions indépendants) afin qu'il devienne un ouvrage de référence. Les commentaires formels sont très positifs: nous avons eu de très bons retours à tous les niveaux.

Quel impact croyez vous que cet ouvrage aura sur la pratique? FM Sur la pratique ce sera avec les années que nous pourrons le voir. Il faudra laisser la possibilité au temps de faire son travail, puisqu'on ne consulte ni ne lit pas, de toute évidence, un ouvrage comme celui-ci d'un couvert à l'autre. Nous avions comme objectif d'offrir un ouvrage de réflexion, afin que des ouvertures puissent se faire: aujourd'hui encore, c'est ce qui nous semble fondamental.

\section{Finalement, la muséologie est-elle pour vous une science appliquée ou une discipline?}

FM Lorsque vous lisez ce dictionnaire, il est évident qu'il s'agit pour moi d'une discipline. Je conçois qu'elle puisse aussi être qualifiée de science appliquée ou autre, mais je le vois comme un faux débat, ce n'est pas cela qui me semble être le plus intéressant. Ce qui est important, c'est que ce soit plus qu'une application pratique, et c'est ce que nous avons tenté de démontrer, qu'il y a une façon élargie de concevoir le muséal, de réfléchir sur le musée. Si cet espace peut être articulé comme une science, ce vocabulaire n'est pas le mien; je préfère parler plutôt d'un ensemble de théories. Nous avons essayé dans cet ouvrage de prendre appui sur tout ce qui a été écrit dans son ensemble: des théories d'une part, de la pratique de l'autre, et non seulement d'en présenter une synthèse (parce que tout ne peut pas être synthétisé), mais aussi d'en baliser les éléments. Il s'agit en fait du principe de la carte: ces 21 concepts représentent une sorte de carte du champ. On peut passer par l'un ou par l'autre, il s'agit toujours de muséologie; et si l'on veut 
comprendre l'ensemble, il est intéressant de prendre appui sur cette vision globale. Nous espérons que l'ouvrage pourra donner de nouveaux points de repère à ceux qui œuvrent dans le milieu pour réfléchir à leur pratique. Personnellement, cela m'a permis de faire le point sur des conceptions, des disciplines qui peuvent être antinomiques, de les présenter et de montrer en quoi elles diffèrent. De montrer qu'il ne s'agit pas de déterminer quelle est la meilleure, mais de comprendre pourquoi elles sont intéressantes, tout en exposant leurs spécificités et en quoi elles peuvent s'enrichir mutuellement. 